\title{
À l'école de la République, de « l'égalité filles / garçons » à la « culture de l'égalité »
}

\section{Muriel Salle}

\section{OpenEdition}

\section{Journals}

Édition électronique

URL : https://journals.openedition.org/trema/3570

DOI : $10.4000 /$ trema.3570

ISSN : 2107-0997

\section{Éditeur}

Faculté d'Éducation de l'université de Montpellier

Édition imprimée

Date de publication : 1 décembre 2016

Pagination : 5-13

ISBN : 979-10-96627-02-8

ISSN : 1167-315X

\section{Référence électronique}

Muriel Salle, «À l'école de la République, de «l'égalité filles / garçons » à la « culture de l'égalité » », Tréma [En ligne], 46 | 2016, mis en ligne le 01 avril 2017, consulté le 21 septembre 2021. URL : http:// journals.openedition.org/trema/3570 ; DOI : https://doi.org/10.4000/trema.3570

Ce document a été généré automatiquement le 21 septembre 2021.

Trema 


\title{
À l'école de la République, de «l'égalité filles / garçons » à la « culture de l'égalité »
}

\author{
Muriel Salle
}

1 Le 25 septembre 2012, Vincent Peillon, ministre de l'Éducation nationale, et Najat Vallaud-Belkacem, en charge des droits des femmes, publient une tribune entendant «mettre l'égalité des filles et des garçons au cœur même de la refondation républicaine de l'école » (Peillon 2012). Résumant efficacement quarante années de recherches en sociologie de l'éducation ce texte, qui souligne que « la manière d'interroger, de donner la parole, de noter, de sanctionner et évidemment d'orienter, révèle des représentations profondément ancrées sur les compétences supposées des unes et des autres ", ouvre une séquence chronologique dense et complexe. Entre cette déclaration d'intentions programmatique publiée quelques mois seulement après l'élection présidentielle, et l'annonce du lancement d'un « plan d'action pour l'égalité entre les filles et les garçons à l'école » assorti de la mise en ligne d'une « mallette pédagogique » pour la rentrée 2014, deux années se sont écoulées, qui ont été marquées par bien des polémiques et quelques revirements. Alors que le concept de «genre" fait irruption sur la scène politique et médiatique, le rôle de l'école en matière de promotion et de diffusion d'une «culture de l'égalité entre les sexes » présentée comme « une valeur centrale et une promesse de la République » est affirmé comme jamais par l'Éducation nationale. Le sujet n'est pourtant pas neuf. Depuis le 26 décembre 1976 et la publication des décrets d'application de la loi Haby du 11 juillet 1975 généralisant les classes mixtes à tous les établissements d'enseignement publics du primaire et du secondaire, les textes officiels, de natures diverses, se sont succédés sur la question. Sur quels principes structurants s'appuient les politiques qui en découlent ? C'est à cette question de recherche que l'on répondra ici en portant une attention toute particulière au vocabulaire mobilisé par l'institution. Après une présentation des différents concepts utilisés, on en viendra à l'analyse des conventions - bilatérales puis interministérielles - qui ont porté sur la question depuis 1984. On pourra ainsi prendre pour finir la mesure des évolutions récentes de la posture ministérielle, consécutive de ce qu'on pourrait appeler « la crise des ABCD de l'Égalité ». 


\section{Quels concepts pour quelle égalité ?}

2 Si elle réfléchit de longue date à la question de l'égalité entre les sexes à l'école, l'Éducation nationale ne s'est jamais préoccupée d'en donner une représentation unifiée (Pasquier 2013). Qu'elle n'ait pas cerné les enjeux d'une prise de position claire sur le sujet, ou qu'elle ait fait sciemment ce choix pour contenter toutes les parties, elle n'a ainsi jamais choisi explicitement entre la perspective différentialiste promotrice d'une " égalité dans la différence » et la perspective universaliste pour laquelle tous les êtres humains sont égaux en dépit de la différence des sexes, qui n'est qu'un rapport de pouvoir. Les expressions consacrées par l'usage institutionnel sont même révélatrices de postures idéologiques variables. Parler de "mixité », d' "égalité entre les filles et les garçons », de "genre » ou de «culture de l'égalité filles/garçons », ça n'est pas la même chose. De la mixité il n'est d'ailleurs presque jamais question.

On sait comment elle s'est imposée dans le courant du XIXe siècle "sans même qu'on y prête attention ", en dépit du fait qu'elle constitue une "révolution pédagogique » parmi les «plus profondes » (Prost 2004, p. 576). La loi Haby du 11 juillet 1975 la généralise pour des raisons pratiques. Il s'agit d'œuvrer non pas «au nom de principes, d'ailleurs passionnément discutés, mais pour servir les familles au plus proche de leur domicile » ainsi que le précise une circulaire ministérielle du 3 juillet 1957. Ainsi envisagée, la mixité scolaire n'en est pas vraiment une. Le regroupement par âge (et non par sexe) des garçons et des filles est un mode d'organisation administrative visant à faire des économies : au sens strict c'est de la gémination (Zancarini 2003, p.12). La coéducation relève quant à elle d'un choix de société et vise à «construire des rapports harmonieux et égalitaires entre les hommes et les femmes" (Zancarini 2003, p.14). Enfin, parler de mixité permet longtemps de rester dans le flou. Ce n'est qu'en 1989 que cette dernière se voit assigner un rôle de promotion de l'égalité entre les sexes ${ }^{1}$. L'expression "égalité entre les filles et les garçons » est récurrente dans la rhétorique institutionnelle mais, là encore, son sens mérite d'être précisé. Le flou qui entoure la notion d'égalité a été souligné récemment par les évaluateurs du dispositif « $\mathrm{ABCD}$ de l'Egalité ». Il apparait nécessaire de "préciser le sens de l'éducation à la valeur égalité, particulièrement quand il s'agit de l'égalité fillesgarçons » (Évaluation 2014). «Il semblerait plus prudent de ne plus utiliser l'expression "l'égalité filles - garçons" sans faire référence au droit » (Évaluation 2014, p.25), écriventils. Il convient donc de parler de «l'égalité des droits des filles et des garçons », ou encore de «l'égalité des compétences » lit-on encore. Adopter l'une ou l'autre de ces expressions pose toutefois question. Si «l'égalité des droits est une notion suffisamment générique » (Évaluation 2014, p. 25), elle s'oppose néanmoins à l'égalité en fait ou égalité réelle, que Najat Vallaud-Belkacem, qui fut ministre des droits des femmes avant d'être en charge de l'Éducation nationale, appelait pourtant de ses vœux à l'occasion du vote de la loi du 4 août 2014. Préconiser l'usage d'une telle expression constitue donc un net recul par rapport aux objectifs initialement affichés. Au prétexte que, pour certains parents, « l'égalité signifie le fait de ne pas reconnaître les différences » (Évaluation 2014, p.17), on réaffirme le principe d'une "égalité dans la différence», un principe politicophilosophique datant du XIXe siècle ${ }^{2}$ et qui «a avant tout pour but de contrer les revendications d'égalité des féministes" (Mosconi, 2012). Quant à parler d'égalité des compétences, c'est souscrire toujours au principe fixiste de la bicatégorisation de sexes et à celui, sous-jacent, de l'existence de « rôles de sexes » envisagés comme complémentaires, 
dans une perspective hétéronormative. La "culture de l'égalité filles/garçons » apparue dans le vocabulaire ministériel à compter de 2013 creuse le même sillon idéologique. On poursuit des perspectives égalitaires tout en posant l'axiome de la « nature singulière » et spécifique des filles versus de celle (tout aussi singulière et spécifique) des garçons. Si l'on souligne que l'égalité relève d'une «culture » qu'il convient d'acquérir et de diffuser, ce qui manifeste d'une vraie prise de conscience et d'un renoncement appréciable à l'illusion de l'égalité, il n'est en revanche pas question de toucher à la "nature ». Le syntagme «culture de l'égalité filles/garçons » laisse entendre le relativisme dans lequel le principe d'égalité des sexes se trouve englué, ce qui l'expose à la fois aux remises en cause et aux instrumentalisations. Conséquence des polémiques que l'on sait (à ce propos voir Fassin 2011, Fillod 2014, Rochefort 2014, Van der Dussen et al. 2015), le concept de genre n'est quant à lui plus utilisé. Pourtant en éducation, il permet de déplacer le regard, des filles vers la conjonction garçons et filles, et de prendre la mesure de la valence différentielle des sexes (Héritier 1996), ce qui n'est encore que partiellement fait. Dans la rhétorique institutionnelle, "élève» demeure souvent un substantif neutre, sauf quand il est spécifiquement décliné au féminin, et ce en dépit de travaux importants (Ayral 2011 et 2014) montrant que l'élève au masculin a des caractéristiques et une destinée scolaire spécifique. Cette même rhétorique ignore encore le plus souvent l'asymétrie qui existe entre filles et garçons, masculin et féminin, peinant du coup à rendre compte, par exemple, les trajectoires professionnelles de celles et ceux qui font le choix d'une orientation contre-stéréotypée et qui, paraissant mues par un même ressort, aboutissent pourtant le plus souvent à des situations diamétralement opposées (réussite éclatante des hommes isolés dans un secteur professionnel réputé féminin versus difficultés accrues rencontrées par les femmes évoluant dans un milieu considéré comme masculin).

4 Le recours au concept de genre implique également un « déplacement de l'attention des sujets - les acteurs, qu'ils soient individus ou groupes sociaux - vers les processus de "genrisation" » qui modèlent et hiérarchisent les individus selon leur sexe (Salle 2013, p. 173). On adopte ainsi une perspective résolument culturaliste permettant d'éviter l'écueil des «remises en question individuelles [qui] peuvent être déstabilisantes", ainsi que le souligne Céline Petrovic (2013, p. 48), au point d'empêcher l'adhésion des participant-es aux formations à l'égalité filles/garçons. Le genre, décliné au seul singulier pour désigner un système, permet de sortir de la bicatégorisation dans laquelle on continue de se complaire quand on lui préfère l' "égalité filles/garçons ». Il ne s'agit bien sûr pas de dire que les filles et les garçons n'existent pas dans nos écoles. Ce ne sont pas ces réalités qui posent problèmes, mais bien les catégories qui y sont associées. On sait les effets du processus même de catégorisation sur les élèves ${ }^{3}$. Adopter une perspective de genre permettrait de rompre avec la spécification qui l'accompagne, et de mettre en œuvre des actions efficaces. Pour parvenir à une égalité réelle entre les groupes que l'on veut égaliser, il faut refuser de reproduire la distinction entre eux et partir, plutôt, de ce qui transcende chacune de ces deux catégories identitaires.

\section{D'une convention à l'autre : les évolutions de la posture ministérielle}

5 Une réflexion officielle sur les enjeux de la mixité des sexes à l'école existe depuis la première circulaire concernant le fonctionnement des établissements mixtes du 3 juillet 1957. L'engagement institutionnel prend toutefois une autre dimension après l'élection 
de François Mitterrand à la présidence de la République. En 1981, Yvette Roudy veut faire de son ministère des droits de la femme celui du « déconditionnement » (Thébaud, 2001) et, si l'on ne parle pas encore de "stéréotypes de genre ", l'arrêté du 12 juillet 1982 consacre la nécessité d'une "Action éducative contre les préjugés sexistes ». La réflexion qui s'amorce alors sur la question de l'égalité entre les sexes est conduite dans une perspective plus économique que sociale et culturelle. Les conventions bilatérales du 20 décembre 1984, puis du 14 septembre 1989, visent essentiellement la diversification des choix professionnels des jeunes filles. Il s'agit de promouvoir l'idée que « les métiers n'ont pas de sexe » auprès du public, en l'occurrence «les jeunes filles et leurs parents ». Au motif « le pays manque d'ingénieurs et de techniciens », campagne est de nouveau menée pour une ouverture des perspectives professionnelles des filles en 1989. Il ne s'agit donc pas d'œuvrer pour une égalité entre les élèves de l'un et l'autre sexe à l'école, mais bien d'encourager la présence de jeunes filles dans des secteurs de formation scientifiques et techniques où elles sont traditionnellement peu nombreuses ${ }^{4}$. Dans le contexte économique du temps, et alors que ces branches professionnelles paraissent être celles par lesquelles la crise pourrait être résorbée, la diversification des choix professionnels féminins est motivée par la poursuite d'une efficacité économique plus que par un quelconque principe de justice sociale. Pour l'institution scolaire, la question de l'égalité des sexes émerge ensuite plus discrètement pendant une décennie. Pourtant, la nécessité d'une prise en compte plus globale et plus ample de la problématique de l'élargissement des choix professionnels des filles est d'ores et déjà mise en avant. L'évaluation des effets des politiques successives de promotion de l'égalité des sexes à l'école est mitigée. Dominique Torsat insiste alors sur la nécessité de «travailler dans une optique plus globale ", d'instaurer une "politique forte pouvant pallier la dispersion des actions et redonner des impulsions créatrices » afin progresser vers une véritable politique d'égalité des sexes (Belloubet-Frier 2011). L'année 2000 voit la signature de la première d'une série de conventions interministérielles pour l'égalité entre filles et garçons, femmes et hommes dans le système éducatif. La nécessité d'une approche globale pour embrasser la problématique a été entendue: une dynamique interministérielle est amorcée qui implique 5 ministères et secrétariats d'État ${ }^{5}$ en $2000,8^{6}$ en 2007 et $6^{7}$ en 2013. Dans leur globalité, les trois textes conventionnels sont semblables, exception faite d'une variation notable du titre dont l'idée d' "égalité des chances » disparaît entre la version de 2000 et celle de 2007. Ils s'organisent tous autour d'un préambule dans lequel le succès scolaire des filles est rappelé ${ }^{8}$, de même que la neutralisation de cet avantage scolaire au moment de l'entrée dans la vie professionnelle. Comme dans le cadre des conventions bilatérales des années 1980, c'est le destin professionnel des filles qui retient essentiellement l'attention. Ainsi, l'» accès inégalitaire des femmes et des hommes au marché du travail » est rappelé dans la convention de 2000. En 2007, c'est la constatation de « la persistance de difficultés rencontrées par les femmes dans leur trajectoire professionnelle » qui est soulignée, cependant qu'en 2013 on insiste sur les «choix d'orientation [féminins qui] demeurent très traditionnels et trop souvent restreints à quelques secteurs d'activité ». Ces variations sur le même thème sont d'ailleurs assez désespérantes, tant ces continuités rédactionnelles laissent présager de persistances des inégalités sur le terrain scolaire et professionnel. Toutefois, ces conventions des années 2000 déploient la question de l'égalité entre les sexes dans des perspectives plus large que précédemment. Certes, il est toujours question d'orientation professionnelle, mais plus seulement de celle des filles. 
6 La convention de 2000 vise ainsi à "améliorer » aussi celle "des garçons ». Surtout, conformément à l'article L 121-1 du Code de l'Éducation, la mixité se voit assigner un rôle de promotion de l'égalité entre les sexes". Il s'agit désormais de "promouvoir une éducation fondée sur le respect mutuel des deux sexes » (Convention 2000), d' « assurer auprès des jeunes une éducation à l'égalité entre les sexes » (Convention 2007), voire d'en profiter pour "acquérir et transmettre une culture de l'égalité entre les sexes » (Convention 2013). D'une version à l'autre de la convention, les grands objectifs poursuivis par le texte ne varient presque pas mais on relève un infléchissement dans leur hiérarchisation: en 2000 et 2007 , la problématique de l'orientation prime celle du " respect mutuel des deux sexes » (convention de 2000) et celle d'une "éducation à l'égalité entre les sexes» (2007), le dernier objectif des textes étant la promotion d' " outils de promotion de l'égalité et de formation» (2000), devenu ensuite "pratiques professionnelles et pédagogiques » (2007).

7 En 2013, c'est d'abord de la nécessité d' " acquérir et transmettre une culture de l'égalité entre les sexes" qu'il est question avant que ne soit évoqué le renforcement de « l'éducation au respect mutuel et à l'égalité entre les filles et les garçons, les femmes et les hommes » et « une plus grande mixité des filières de formation et à tous les niveaux d'étude ». La problématique de l'orientation se trouve ainsi reléguée en troisième et dernière position. Ces variations d'un texte à l'autre sont certes subtiles, mais certainement révélatrices. En effet, on ne fait pas la même chose quand on veut " promouvoir une éducation fondée sur le respect mutuel des deux sexes ${ }^{10}(2000)$, quand on entend " assurer une éducation à l'égalité entre les sexes" (2007) et quand on cherche à " acquérir et transmettre une culture de l'égalité entre les sexes» (2013). Si en 2000 le respect entre les deux sexes était fondateur de l'éducation, et pouvait donc passer pour un prérequis de toute posture éducative, la convention de 2007 manifeste une relative prise de conscience. L'égalité entre les sexes n'a rien de spontané. Il convient d'y être éduqué. Ce faisant, l'égalité des sexes n'est plus un principe au sens épistémologique du terme, une proposition directrice. Elle relève de ces « objets à l'identité curriculaire floue qui échappent à la classification traditionnelle des savoirs et qu'on désigne généralement sous le terme vague d'“éducation à" " (Forquin 2003, p.128). Gaël Pasquier rappelle que leur enseignement ne va pas de soi dans les sociétés démocratiques contemporaines parce qu'ils « entendent propager une définition de ce qui est juste et bien indépendamment du choix des familles»(2010, p.69). Le recours en dernière intention à l'expression de "culture de l'égalité » poursuit dans la même veine, entre réalisme bienvenu et fragilisation collatérale. Parler en ces termes c'est admettre que l'égalité entre les sexes est un objectif éducatif discuté, dont le contenu - variable - doit être précisé, dont les attendus méritent d'être définis, et dont les modalités - changeantes - doivent être spécifiées. Avec ces glissements de sens, d'une convention à l'autre, on passe de l'affirmation d'un principe fondateur qui peut, certes, demeurer incantatoire mais n'en est pas moins ferme à une culture de qui pèche au mieux par sa parfaite inocuité, au pire par son complet relativisme. Le vocabulaire qui se déploie dans chacune des conventions témoigne, lui aussi, de subtiles évolutions. Le champ lexical des problématiques d'égalité est vaste, ce qui autorise des variations révélatrices. Entre 2000 et 2013, la mention du sexisme est de plus en plus assumée : l'adjectif « sexiste » apparaît 4 fois dans le texte de 2000, 20 fois en 2013. De même, la notion de «stéréotype » qui devient un incontournable dans le texte de 2013 (17 mentions contre 3 en 2000). La « sexualité », quelle qu'elle soit, est en revanche un angle mort de ces conventions : le terme n'apparaît ni en 2000, ni en 
2013. En 2007, il est fait mention une seule fois de l'» éducation à la sexualité ». Quant au " genre », complètement absent de la rhétorique ministérielle en 2000, il s'impose en 2013 ( 9 mentions) après une très timide poussée en 2007 (une mention). Il est notable toutefois qu'aucun texte ministériel ne prenne la peine (ou le risque) d'en donner une définition. Si «les savoirs scientifiques issus des recherche sur le genre [...] doivent nourrir les politiques publiques » (Convention 2013), nul ne dit ce dont il s'agit au juste. Et il y a fort à parier que le terme joue ici «le rôle d'un label» (Chaperon 2003), une étiquette commode mais, hélas, vidée de son sens, en l'occurrence de sa dimension critique.

La réflexion institutionnelle sur l'égalité des sexes à l'école est ancienne mais les polémiques récentes à laquelle elle a été confrontée, en lien notamment avec la mise en œuvre du dispositif « $A B C D$ de l'Égalité » ont permis de faire émerger un questionnement aigu: quelle égalité entend-on exactement promouvoir à l'école de la République ? L'évolution de la rhétorique ministérielle, lisible dans les conventions analysées ici le montre bien. Dans les années 1980, la différence des sexes est pensée sur le mode de l'asymétrie: seul le destin scolaire et professionnel des filles et des femmes intéresse chercheures et politiques. Par la suite, la symétrie filles/garçons s'impose, accompagnée de l'invisibilisation du système de genre et de la valence différentielle qui y préside. Si la convention de 2013 se réfère au concept de "genre ", après les polémiques de l'année 2013-2014, l'égalité promue entre filles et garçons relève d'une perspective résolument différentialiste (Buscatto et al. 2015) qui s'exprime dans le recours au syntagme désormais en usage de «culture de l'égalité filles/garçons". Penser en terme de genre paraît pourtant indispensable si l'on veut résoudre les défis éducatifs qui se posent à l'école d'aujourd'hui. On ne peut en faire l'économie si l'on veut comprendre que c'est ce système qui explique l'avantage scolaire des filles mais aussi leur insertion et leur réussite professionnelles mitigées par la suite. Il est indispensable pour analyser, et tenter de pallier, l'échec scolaire de certains garçons. Il permet de sortir d'une approche dirigée spécifiquement vers les filles, approche à la fois inutile (puisque les filles réussissent mieux à l'école que les garçons), inefficace (puisque cet avantage scolaire reste sans conséquence dans le cadre professionnel) et contre-productive (puisqu'elle contribue à décrédibiliser des actions considérées comme catégorielles). Il ne s'agit pas de faire des filles des élèves comme les garçons, ni des garçons comme les filles, mais de faire de chacun et chacune un-e élève comme les autres. Nul ne songe à contester l'existence d'une différence anatomique entre hommes et femmes, mais ce qui est en cause et à quoi il ne faut pas renoncer, c'est en revanche l'idée que l'anatomie puisse être un destin. Les rôles sociaux incombant aux filles comme aux garçons doivent être appréhendés comme des constructions culturelles et historiques, variables dans le temps comme dans l'espace. Voilà sans doute le sujet sensible auquel la formation des enseignant-es conçue en termes de « culture de l'égalité filles/garçons » ne s'attaque pas.

\section{BIBLIOGRAPHIE}




\section{Sources et textes officiels par ordre chronologique de parution}

Convention de 1984. En annexe du Rapport d'information sur l'égalité professionnelle entre les femmes et les hommes présenté par Yvette Roudy à l'Assemblée nationale lors de la séance du 20 décembre 1984. http://www.assemblee-nationale.fr/histoire/femmes/r1661roudy.pdf [Consulté le 20/10/2015]

Convention de 1989.

Convention de 2000. Bulletin officiel $\mathrm{n}^{\circ} 10 \mathrm{du} 9$ mars 2000, «Convention pour la promotion de l'égalité des chances entre les filles et les garçons, les femmes et les hommes, dans le système éducatif ». http://www.education.gouv.fr/bo/2000/10/orga.htm [Consulté le 20/10/2015]

Convention de 2007. Bulletin officiel $n^{\circ} 5$ du 1er février 2007, «Convention pour l'égalité entre les filles et les garçons, les femmes et les hommes, dans le système éducatif (2007-2013) ». http:// www.education.gouv.fr/bo/2007/5/MENE0603248X.htm [Consulté le 20/10/2015]

Peillon (Vincent) et Vallaud-Belkacem (Najat), « Pour plus d'égalité entre filles et garçons à l'école ", in Le Monde, 25 septembre 2012. http://www.lemonde.fr/idees/article/2012/09/25/ pour-plus-d-egalite-entre-filles-et-garcons-a-l-ecole_1765510_3232.html [Consulté le 20/10/2015]

Convention de 2013. Bulletin officiel $n^{\circ} 6$ du 6 février 2013, «Convention pour l'égalité entre les filles et les garçons, les femmes et les hommes, dans le système éducatif (2013-2017) ». http:// www.education.gouv.fr/pid25535/bulletin_officiel.htmletcid_bo=67018 [Consulté le 20/10/2015]

Évaluation du dispositif « ABCD de l'Egalité » Rapport n²014-047, juillet 2014. http:// www.education.gouv.fr/cid80894/evaluation-du-dispositif-experimental-abcd-de-l-egalite.html [Consulté le 20/10/2015]

\section{Bibliographie}

Ayral, S. (2011), La fabrique des garçons : sanctions et genre au collège. Paris : PUF.

Ayral, S. et Raibaud Y. (dir.) (2014). Pour en finir avec la fabrique des garçons. Pessac : MSHA, 2014.

Belloubet-Frier, N.(2004). «L'égalité des sexes dans le système éducatif : une dynamique interministérielle en construction ». Colloque « De la mixité à l'égalité dans le système éducatif » du 27 mai 2004. http://eduscol.education.fr/cid45879/l-egalite-des-sexes-dans-le-systemeeducatif\%C2\%A0-une-dynamique-interministerielle-en-construction.html [Consulté le 20/10/2015]

Buscatto, M. ; Chevalier Y. et Collet I. et al., «Égalité des sexes à l'école : machine arrière, toute !». Médiapart, 16 janvier 2015. http://blogs.mediapart.fr/edition/les-batailles-de-legalite/ article/160115/egalite-des-sexes-1-ecole-machine-arriere-toute [Consulté le 20 octobre 2015] Chapero, S. (2003). « Le genre : un mot, un concept ou un label ? ", in Fougeyrollas-Schwebel D. ; Planté, C.; Riot-Sarcey, M. et Zaidman, C. Le genre comme catégorie d'analyse. Sociologie, histoire, littérature. Paris : l'Harmattan.

Fassin, E. et Margron, V. (2011). Homme, femme, quelle différence ? La théorie du genre en débat. Paris : Éditions Salvator. 
Fillod, O. (2014). «L'invention de la "théorie du genre" : le mariage blanc du Vatican et de la science ", in Contemporary French Civilization, vol. 39, $\mathrm{n}^{\circ} 3$.

Forquin, J-C. (2003). « La critique communautarienne du libéralisme politique et ses implications possibles pour l'éducation ", Revue française de pédagogie, $\mathrm{n}^{\circ} 413$.

Héritier, F. (1996). Masculin, Féminin. La pensée de la différence. Paris : Odile Jacob.

Mosconi, N. (2012), Henri Marion et « l'égalité dans la différence », Le Télémaque, N41.

Pasquier, G. (2010). « Enseigner l'égalité des sexes à l'école primaire », Nouvelles Questions Féministes, Vol.29.

Pasquier, G. (2013). Les pratiques enseignantes en faveur de l'égalité des sexes et des sexualités à l'école primaire, vers un nouvel élément du curriculum. Thèse de doctorat : Université Paris Ouest Nanterre La Défense.

Petrovic, C. (2013), « La formation des enseignant-e-s et le genre : quelles résistances ? », Formation et pratiques d'enseignement en question, $\mathrm{n}^{\circ} 16$.

Prost, A. (2014). Histoire générale de l'enseignement et de l'éducation en France, Tome IV : L'école et la famille dans une société en mutation, depuis 1930. Paris : Perrin, 2004. Rochefort, F.,

" "Mariage pour tous" : genre, religions et sécularisation », in Laufer, L. et Rochefort, F. Qu'est-ce que le genre? Paris : Payot.

Salle, M. et Gallot, F. (2013). « Femmes ? Genre ? Mixité ? Quelles nouvelles perspectives pour l'enseignement de l'histoire ?", in Morin-Messabel, C. et Salle, M., À l'école des stéréotypes. Comprendre et déconstruire. Paris : L'Harmattan.

Thébaud F. (2001). « Promouvoir les droits des femmes : ambitions, difficultés et résultats ", dans Berstein, S., Milza, P. et Bianco, J-L. (Ed.), Les années Mitterrand : les années du changement. Paris : Perrin.

Van der Dussen, S. ; Paternotte, D. et Piette, V. (2015). Habemus gender ! Déconstruction d'une riposte religieuse. Bruxelles : Editions de l'Université.

Zancarini-Fournel, M. et Thébaud, F.(2003). « Éditorial », Clio. Histoire, femmes et sociétés, n 18.

\section{NOTES}

1. La loi Jospin de 1989 précise : «les écoles, les collèges, les lycées et les établissements d'enseignement supérieur contribuent à favoriser l'égalité entre les hommes et les femmes». Ce principe, intégré au Code de l'Education est formulé dans l'article L.121 : « les écoles, les collèges, les lycées et les établissements d'enseignement supérieur [...] contribuent à favoriser la mixité et l'égalité entre les hommes et les femmes, notamment en matière d'orientation ".

2. Le principe de l' «égalité dans la différence » est inventé par Ernest Legouvé qui, au printemps 1848, consacre un cours au Collège de France à ce thème avant de le développer dans un livre intitulé Histoire morale des femmes.

3. Sur la sociologie de l'étiquetage, voir les travaux de E. M. Lemert, E. Goffman et H. Becker.

4. Le prix de la vocation scientifique et technique est institué en 1991, en remplacement de la bourse du même nom instaurée en 1985 .

5. En 2000, sont signataires de la convention la ministre de l'emploi et de la solidarité, le ministre de l'éducation nationale, de la recherche et de la technologie, le ministre de l'agriculture et de la pêche, la ministre déléguée chargée de l'enseignement scolaire, et la secrétaire d'État aux droits des femmes et à la formation professionnelle. 
6. Le ministère de l'emploi, de la cohésion sociale et du logement, le ministère de l'Éducation nationale, de l'enseignement supérieur et de la recherche, le ministère de la justice, le ministère des transports, de l'équipement, du tourisme et de la mer, le ministère de l'agriculture et de la pêche, le ministère de la culture et de la communication, le ministère délégué à la cohésion sociale et à la parité, le ministère délégué à l'enseignement supérieur et à la recherche.

7. Le ministère de l'éducation nationale, le ministère délégué chargé de la réussite éducative, le ministère des droits des femmes, le ministère du travail, de l'emploi, de la formation professionnelle et du dialogue social, le ministère de l'enseignement supérieur et de la recherche et le ministère de l'agriculture, de l'agroalimentaire et de la forêt.

8. «...la réussite scolaire des filles est aujourd'hui incontestable» en 2000. "Aujourd'hui, les femmes poursuivent des scolarités jusqu'au plus haut niveau de formation » en 2007. « ...les filles ont de meilleurs résultats scolaires que les garçons » en 2013.

9. La loi Jospin de 1989 précise: «les écoles, les collèges, les lycées et les établissements d'enseignement supérieur contribuent à favoriser l'égalité entre les hommes et les femmes». Ce principe, intégré au Code de l'Éducation est formulé dans l'article L.121 : « les écoles, les collèges, les lycées et les établissements d'enseignement supérieur [...] contribuent à favoriser la mixité et l'égalité entre les hommes et les femmes, notamment en matière d'orientation ».

10. C'est moi qui souligne.

\section{RÉSUMÉS}

Depuis les années 1980 l'intérêt du ministère de l'Éducation nationale pour la problématique de l'égalité filles/garçons s'est notamment manifesté par la signature d'une série de textes visant à promouvoir l'égalité entre les sexes dans le cadre de l'institution scolaire. L'analyse de ces conventions - bilatérales puis interministérielles - depuis 1984 permet de prendre la mesure des évolutions de la posture ministérielle sur le sujet. Qu'est-ce, finalement, que la «culture de l'égalité filles/garçons " désormais promue par le ministère ? Quelle représentation de l'égalité des sexes à l'école traduit-elle?

Since the 1980's, the Ministry of Education is interested in the issue of equality between girls and boys. He mainly published several texts to promote gender equality within the school institution. The analysis of these agreements since 1984, allows to measure the evolutions of the ministerial position on the subject. What is the "culture of equality between girls and boys" which is now promoted by the department? What representation of gender equality in school does it reflect?

\section{INDEX}

Mots-clés : égalité, mixité, genre, culture de l'égalité filles/garçons, école

Keywords : equality, co-education, gender, culture of equality between girls and boys, school 


\section{AUTEUR}

MURIEL SALLE

Maîtresse de Conférences - Université Claude Bernard Lyon ESPE de Lyon, Laboratoire de Recherche Historique en Rhône Alpes (LARHRA) 